Pacific Journal of Mathematic 


\title{
ACTIONS OF FINITE GROUPS ON KNOT COMPLEMENTS
}

\author{
FeNG LUO
}

\begin{abstract}
We examine the symmetry of the complement of a non-trivial knot $K$ in $S^{3}$ and obtain a classification of the actions of finite groups on the complement of a non-trivial knot in the case where the actions extend to non-fixed point free actions on the three sphere. We prove the result by showing first an extension theorem which says that any action of finite group on a non-trivial knot complement extends to an action on the three sphere and then by applying the solution of the Smith conjecture.
\end{abstract}

Let $N(K)$ be a regular neighborhood of $K ; m, l$ be a meridian and a preferred longitude of $K$ in $\partial N(K)$ respectively; [m], [l] be the homology classes in $H_{1}(\partial N(K), Z)$ represented by $m, l$ respectively. A knot is called a hyperbolic knot if $S^{3}-K$ has a hyperbolic structure. See $[R]$, or $[B, Z]$ for the standard terminology that we use. The main results of this note are the following. Theorem 1 also follows from the recent result of Gordon and Luecke $[\mathbf{G}, \mathbf{L}]$. Since the proof is simple, it is included here for completeness.

THEOREM 1. If $K$ is a hyperbolic knot, then any self-diffeomorphism of the knot complement $S^{3}-\operatorname{int}(N(K))$ extends to a self-diffeomorphism of $S^{3}$.

Satellite knots have property $\mathbf{P}$ by Gabai's work, and torus knots are also known to have property P. One obtains the following theorem.

COROllary 1. Any self-diffeomorphism of a non-trivial knot complement $S^{3}-N(K)$ extends to a self-diffeomorphism of $S^{3}$.

THEOREM 2. If $G$ is a finite group acting smoothly on the complement $S^{3}-\operatorname{int}(N(K))$ of a non-trivial knot $K$, then the group $G$ is a cyclic or a dihedral group, and the G-action extends to a G-action on $S^{3}$. In particular, if $K$ is a hyperbolic knot, then $\operatorname{Out}\left(\pi_{1}\left(S^{3}-K\right)\right)$ (or what is the same $\left.\operatorname{Isom}\left(S^{3}-K\right)\right)$ is a cyclic or a dihedral group.

With the help of a computer, Riley [Ri] has calculated the 
$\operatorname{Out}\left(\pi_{1}\left(S^{3}-K\right)\right)$ for the following hyperbolic knots, $5_{2}, 6_{3}, 7_{7}, 8_{21}$, $9_{35}, 9_{43}$, and $9_{48}$, the corresponding groups are: $D_{2}, D_{4}, D_{4}, D_{2}$, $D_{6}, Z_{2}$, and $D_{6}$. The theorem explains the general fact behind Riley's work. Combining with the work of Culler, Gordon, Luecke, Shalen (see [CGLS]), Bleiler and Scharlemann $[\mathbf{B}, \mathbf{S}]$ on the property $\mathbf{P}$ of non-trivial knots invariant under non-trivial periodic automorphisms of $S^{3}$, we have the following.

COROLlARY 2. If there exists a finite group acting smoothly nontrivially on a knot complement in $S^{3}$, then the knot has property $\mathrm{P}$. In particular, if $K$ is a hyperbolic knot with non-trivial $\operatorname{Out}\left(\pi_{1}\left(S^{3}-K\right)\right)$, then $K$ has property $\mathrm{P}$.

If the group $G$ in Theorem 2 is cyclic, the $G$-action on the knot complement can be described more explicitly. Before stating the corollary, let us make the following conventions. A $2 \pi / n$-rotation of $S^{3}$ is a $Z_{n}$-action which is conjugate to the orientation preserving $Z_{n^{-}}$ action generated by $A$ where $A$ sends a point $(x, z)$ in $S^{3}=R^{1} \times$ $C \cup\{$ infinity $\}$ to $\left(x, e^{2 \pi i / n} z\right)$ and infinity to infinity. The circle $\{(x, z) \mid z=0\} \cup\{$ infinity $\}$ is called the axis of the rotation. A twisted $2 \pi / n$-rotation of $S^{3}$ is an action conjugate to the non-orientation preserving $Z_{n}$-action generated by $\alpha$, where $\alpha$ is described as follows. Represent $S^{3}$ as $\left(R^{1} \times C\right) \cup\{$ infinity $\}, \alpha$ is the automorphism sending $(x, z)$ to $\left(-x,-e^{2 \pi i / n} z\right)$, and infinity to infinity. The circle $\{(x, z) \mid z=0\} \cup\{$ infinity $\}$ is called the axis of the twisted rotation. A reflection of $S^{3}$ through two points is an action conjugate to the orientation reversing involution of $S^{3}$ generated by $\beta$, where $\beta$ is the automorphism of $S^{3}$ considered as $R^{3} \cup$ \{infinity\} sending $x$ to $-x$, for $x$ in $R^{3}$, and infinity to infinity.

COROLlARY 3. The smooth action of a cyclic group $Z_{n}$ on a nontrivial knot complement $S^{3}-\operatorname{int}(N(K))$ are classified as follows.

(I) The action preserves the orientation. There are two cases.

(a) The action on $S^{3}-\operatorname{int}(N(K))$ is free. Then the action is induced by a fixed point free $Z_{n}$-action on $S^{3} . K$ is invariant under the action.

(b) The action is not free. Then the $Z_{n}$-action is induced by a $2 \pi / n$ rotation of $S^{3}$ about a trivial knot $L . K$ is invariant under the rotation. $K$ is disjoint from $L$, or $K$ intersects $L$ transversely in two points. If the latter happens, $n=2$.

(II) The $Z_{n}$-action on $S^{3}-\operatorname{int}(N(K))$ does not preserve the orientation. Then the $Z_{n}$-action has fixed points in $S^{3}$, and is of even order. 
There are four kinds:

(c) $n=2$. Then the action is induced by a reflection $R$ of $S^{3}$ through two points, or is induced by a reflection $R^{\prime}$ of $S^{3}$ with respect to a two-sphere, which is the same as a twisted $\pi$-rotation of $S^{3} . K$ is invariant under the involution. There are three types of $Z_{2}$-actions on $S^{3}-\operatorname{int}(N(K))$.

(c) ${ }_{1} K$ is disjoint from the two fixed points of the reflection $R$. In this case the $Z_{2}$-action on $S^{3}-\operatorname{int}(N(K))$ has two fixed points.

(c) ${ }_{2} K$ contains the two fixed points of $R$. In this case, the $Z_{2^{-}}$ action is a free action on $S^{3}-\operatorname{int}(N(K))$.

(c) ${ }_{3} K$ intersects the 2-sphere fixed points of $R^{\prime}$ transversely in two points. In this case, $K$ is of the form $K=L \#(-L)$ for some knot $L$.

(d) $n \geq 4$. Then the action is induced by a twisted $2 \pi / n$-rotation of $S^{3}$ about an axis $L . K$ is invariant, and is disjoint from $L$.

We state the following as a corollary for convenience.

COROLlARY 4. If a cyclic group $Z_{n}$ generated by $g$ acts smoothly on a non-trivial knot complement $S^{3}-\operatorname{int}(N(K))$ such that $g_{*}([l])=-[l]$ in $H_{1}(\partial N(K), Z)$, then $g$ is an involution.

Combining Corollaries 3 and 4, smooth action of dihedral groups on a knot complement can also be classified. We omit it here.

Recall that a knot $K$ is invertible if $K$ is oriented equivalent to $-K$, the inverted knot of $K ; K$ is amphicheiral if $K$ is equivalent to its mirror-image $K^{*}$.

CoROllaRY 5. If $K$ is a hyperbolic knot in $S^{3}$, then the following holds.

(a) $K$ is invertible if and only if $K$ is invariant under a $\pi$-rotation in $S^{3}$ about an axis $L$ such that $L$ intersects $K$ transversely in two points.

(b) $K$ is amphicheiral if and only if $K$ is invariant under a twisted $2 \pi / n$-rotation of $S^{3}$ about an axis missing $K$, for $n \geq 4$, or $K$ is invariant under a reflection of $S^{3}$ through two points missing $K$.

(c) If $K$ is both invertible and amphicheiral, then $K$ is invariant under a reflection of $S^{3}$ through two points contained in $K$.

In $\S 1$, we prove Theorem 1 . In $\S 2$, we prove Theorem 2 , and its corollaries. In the appendix, we prove the following proposition concerning smooth non-orientation preserving cyclic group actions on $S^{3}$. 
Proposition. Any smooth non-orientation preserving cyclic group action on the 3-sphere is conjugate to a twisted rotation or a reflection of the sphere through two points.

Acknowledgment. The author would like to thank his thesis advisor M. Freedman, and X.-S. Lin for many discussions on knot theory. He also thanks the referee for the comments and for pointing out that Corollary 5(a) was a result of Kawauchi [Ka].

1. Proof of Theorem 1. Let $K$ be a hyperbolic knot in $S^{3}$ with $S^{3}-K$ having a hyperbolic metric; $N(K)$ be a regular neighborhood of $K$ such that $\partial N(K)$ is a flat torus in $S^{3}-K$ with respect to the hyperbolic metric; $m, l$ be a meridian and a preferred longitude of $K$ respectively, $m, l$ lie in $\partial N(K)$ and be realized as geodesics. $m, l$ will also be used to denote the elements in $\pi_{1}\left(S^{3}-\operatorname{int}(N(K))\right)$ represented by them. Let $[m],[l]$ be the homology classes in $H_{1}(\partial N(K), Z)$ represented by $m, l$ respectively. Let $h$ be a self-diffeomorphism of $S^{3}-\operatorname{int}(N(K))$. Our goal is to prove that $h^{*}([m])$ is $\pm[m]$ in $H_{1}(\partial N(K), Z)$. Since if this condition is satisfied,

$$
\left.h\right|_{\partial N(K)}: \partial N(K) \rightarrow \partial N(K)
$$

extends to be a self-diffeomorphism of $N(K)$ which in turn gives an extension of $h$ to $S^{3}$ by gluing. By Mostow Rigidity, one can assume that $h$ is a hyperbolic isometry. $h_{*}([l])=\varepsilon_{1}[l]$ with $\varepsilon_{1}$ being \pm 1 in $H_{1}(\partial N(K), Z)$, because $\pm[l]$ are the only primitive homology classes in $H_{1}(\partial N(K), Z)$ which vanish in $H_{1}\left(S^{3}-\operatorname{int}(N(K)), Z\right)$ under the inclusion homomorphism. $h_{*}$ is an automorphism of $H_{1}(\partial N(K), Z)$; hence $h_{*}[m]=\varepsilon[m]+a[l]$, where $\varepsilon_{2}= \pm 1$, and $a$ is in $Z$. Our goal is to show $a=0$. If $\varepsilon_{1}=\varepsilon_{2}$, i.e., $h$ is orientation preserving, the result is trivial because on one hand $h$, being an isometry of a hyperbolic manifold of finite volume, is of finite order (i.e., composition of $h$ finite times is the identity map; see [M, B], or [Th]), on the other hand the matrix $\left[\begin{array}{cc}\varepsilon_{1} & a \\ 0 & \varepsilon_{2}\end{array}\right]$ has infinite order if $a$ is non-zero. Therefore, we need only to consider the case where $\varepsilon_{1}=-\varepsilon_{2}$. Suppose conversely $a \neq 0$. Then by Culler, Gordon, Luecke, Shalen [CGLS], one has that $a= \pm 1$, and that $K$ does not have property P. Since the matrix $\left[\begin{array}{cc}\varepsilon_{1} & a \\ 0 & \varepsilon_{2}\end{array}\right]$ is of order two, $h_{*} h_{*}=$ id in $H_{1}(\partial N(K), Z)$. Consider the orientation preserving isometry $g=h \circ h . g$ is of finite order; hence it generates a finite cyclic group $G$ acting isometrically on the flat torus $\partial N(K)$. Because $g_{*}([m])=[m]$ and $g_{*}[l]=[l]$ in $H_{1}(\partial N(K), Z), G$ preserves the foliations $\partial N(K)$ by geodesic 
meridians and by geodesic longitudes. The following lemma shows that the $G$-action on $\partial N(K)$ can be extended to a $G$-action on $N(K)$.

LEMMA 1. If $G$ acts isometrically on a flat boundary $\partial N$ of a solid torus $N$ and $g_{*}[m]= \pm[m], g_{*}[l]= \pm[l]$ in $H_{1}(\partial N, Z)$ where $g$ is a generator of $G, m, l$ are a meridian and a longitude of $\partial N$ respectively, then the G-action can be extended to an action on $N$. Moreover the extended $G$-action on the core of $N$ preserves a flat Riemannian metric on it.

Proof. Parametrize $\partial N$ by $(u, v)$, where $u, v$ are the unit complex numbers such that $S^{1} \times\{v\}$ and $\{u\} \times S^{1}$ correspond to the geodesic meridian $m$ and the geodesic longitude $l$ in $\partial N$. Since the action on the homology group $H_{1}(\partial N, Z)$ satisfies the conditions above, the $G$-action on $\partial N$ corresponds now to a $G$-action on $S^{1} \times S^{1}$ preserving the standard product metric and the product structure. Extending the $G$-action on $\partial N$ to $N$ is the same as extending the $G$-action on $S^{1} \times S^{1}$ to $D^{2} \times S^{1}$. The extension of the latter is trivial. To see this, for $g \in G$, we have,

$$
g(u, v)=(\phi(u, g), \psi(v, g))
$$

where $u, v \in S^{1}, \phi(u, g)=\alpha u$, or $\alpha \bar{u}$, and $\psi(v, g)=\beta v$ or $\beta \bar{v}$, for some roots of unity $\alpha$ and $\beta$. The extension of the $G$-action to $D^{2} \times S^{1}$ is given by the same formula with $u$ in $D^{2}=\{z \in C|| z \mid \leq 1\}$. The extended $G$-action still preserves the product metric and acts on the core $\{0\} \times S^{1}$ isometrically with respect to the flat metric induced from $D^{2} \times S^{1}$.

We have now a cyclic group $G$ which acts on $S^{3}$ preserving $K$. If $G$ is non-trivial, then $K$ has property $\mathrm{P}$ by Corollary 7 of Culler, Gordon, Luecke, Shalen [CGLS] which contradicts $a \neq 0$. Therefore $h \circ h=$ id in $S^{3}-\operatorname{int}(N(K))$. It is easy to check, using $a= \pm 1$, $h_{*}([m])=-\varepsilon_{1}[m]+a[l]$ and $h_{*}([l])=\varepsilon_{1}[l]$, that

$$
h_{*}\left(-2 \varepsilon_{1} a[m]+[l]\right)=-\varepsilon_{1}\left(-2 \varepsilon_{1} a[m]+[l]\right) \text {. }
$$

Note that $[l]$, and $-2 \varepsilon_{1} a[m]+[l]$ are primitive elements, and are the $( \pm 1)$-eigenvectors of $h_{*}$ in $H_{1}(\partial N(K), Z)$. The algebraic intersection number of $[l]$ and $-2 \varepsilon_{1} a[m]+[l]$ is \pm 2 . The following lemma shows that $h$ has fixed points in $\partial N(K)$. 
LEMMA 2. Suppose $h$ is an orientation reversing fixed point free involution of a torus $T^{2}$, then the $( \pm 1)$-eigenspaces of $h_{*}$ are generated by two primitive classes with \pm 1 as their algebraic intersection number.

Proof. Since any orientation reversing fixed point free involution of $T^{2}$ has the quotient space homeomorphic to the Klein bottle, and since the Klein bottle has only one orientable two-fold cover up to covering equivalence, any two orientation reversing fixed point free involutions on $T^{2}$ are conjugate. Because the hypothesis and the conclusion of the lemma are invariant under conjugation, the lemma follows by checking a concrete example. Take $T^{2}$ to be $S^{1} \times S^{1}$ parametrized by $(u, v)$, where $u, v \in S^{1}$, the unit circle in the complex plane. Let $h: T^{2} \rightarrow T^{2}$ be the automorphism sending $(u, v)$ to $(\bar{u},-v) . h$ generates a fixed point free orientation reversing involution of $T^{2}$. The 1-eigenspace of $h_{*}$ is generated by the homology class of the curve $\{1\} \times S^{1}$, and the (-1)-eigenspace of $h_{*}$ is generated by the homology class of the curve $S^{1} \times\{1\}$. Hence the algebraic intersection number of the primitive generators of $( \pm 1)$-eigenspaces is \pm 1 .

By the lemma, $h$ has fixed points in $\partial N(K)$. However, $h$ is an orientation reversing involution, $\operatorname{Fix}\left(\left.h\right|_{\partial N(K)}\right)$ is a 1-dimensional submanifold. This implies that $\operatorname{Fix}(h)$ contains a 2-manifold, say $F$. We claim that this is impossible. By Smith theory (see [B], Theorem 5.1), for the $Z_{2}$-action generated by $h$ on the 1-dimensional $Z_{2}$-homology sphere $S^{3}-\operatorname{int}(N(K))$, the fixed point set $\operatorname{Fix}(h)$ is a $Z_{2}$-homology sphere of dimension at most one. Hence $\operatorname{Fix}(h)(=F)$ is an annulus or a Möbius band.

Case 1. $F$ is an annulus. Since $S^{3}-K$ has a hyperbolic structure, $S^{3}=\operatorname{int}(N(K))$ is annulus free. Hence $F$ is parallel to an annulus in $\partial N(K)$. In particular, $F$ is separating. The two components of the complement of $F$ in $S^{3}-\operatorname{int}(N(K))$ are interchanged by $h$ and hence are homeomorphic. Therefore both of them are solid tori. This implies that $S^{3}-\operatorname{int}(N(K))$ is the union of two solid tori along an annulus in their boundaries which contradicts the existence of the hyperbolic structure of finite volume in $S^{3}-K$.

Case 2. $F$ is a Möbius band. $\partial F$ is now a simple closed curve in $\partial N(K)$ fixed by $h$, and hence [ $\partial F]$ is in the 1-eigenspace of $h_{*}$ which is generated by $[l]$, or by $2 a[m]+[l]$ according to $\varepsilon_{1}=1$, or -1 . Thus $\partial F$ and $K$ bound an annulus $A$ in $N(K)$. The Möbius band $F \cup_{\partial} A$ in $S^{3}$ has $K$ as its boundary. Let $L$ be the core of 
the Möbius band. If $L$ is non-trivial, $K$ is the cable knot of $L$. This contradicts that $K$ is a hyperbolic knot. If $L$ is the trivial knot, then $K$ is the $(2, n)$-torus which is again absurd.

This completes the proof of Theorem 1 .

Since any non-trivial knot with property $\mathrm{P}$ has the property that any self-diffeomorphism of the knot complement preserves the meridian, and since the only non-trivial knots which are not known to have property $\mathrm{P}$ are some hyperbolic knots by the work of Gabai and others, Corollary 2 follows from Theorem 1.

2. Proof of Theorem 2. We shall still use the same notations introduced in $\S 1$. Hence $K$ is a non-trivial knot in $S^{3} ; N(K)$ is a regular neighborhood of $K ; m, l$ are a meridian and a preferred longitude of $K$ respectively. $m, l$ lie in $\partial N(K)$. Our first observation is that there exists a flat metric on $\partial N(K)$ such that $G$ acts on $\partial N(K)$ isometrically. This follows from the Geometrization Theorem that any action of a finite group $G$ on a 2-manifold is equivalent to a geometric group action (see $[\mathbf{E}])$. Fix the metric on $\partial N(K)$, and realize $m, l$ by geodesics in $\partial N(K)$. Theorem 1 shows that the $G$-action on $\partial N(K)$ preserves the geodesic meridians and geodesic longitudes in $\partial N(K)$. By Lemma 1 , the $G$-action on $\partial N(K)$ extends to a $G$-action on $N(K)$ such that the extended $G$-action preserves a flat metric on $K$. Hence the $G$-action on $S^{3}-\operatorname{int}(N(K))$ extends to a $G$-action on $S^{3}$ which preserves $K$ and acts on $K$ preserving a flat metric $d$. The restriction of the $G$-action to $K$ gives a representation:

$$
\sigma: G \rightarrow \operatorname{Isom}(K, d) .
$$

The solution of the Smith Conjecture shows that $\sigma$ is a monomorphism. To see this, let $h \in \operatorname{ker}(\sigma)$, and $H$ be the cyclic group by $h$. Then $H$ acts on $S^{3}$ with fixed point set containing $K$, and $H$ preserves each geodesic meridian in $\partial N(K)$. Moreover, $h_{*}([l])=[l]$ in $H_{1}(\partial N(K), Z)$. There are now two cases that might happen.

Case 1. $h_{*}([m])=[m] . h$ is now an orientation preserving homeomorphism because $h_{*}([l])=[l]$ and $h_{*}([m])=[m]$ imply that $h$ is an orientation preserving homeomorphism in $H_{1}(\partial N(K), Z)$. Therefore the $H$-action on a geodesic meridian $m$ is a rotation. Suppose $h \neq \mathrm{id}$; then $H$ acts non-trivially on $m$. Therefore $K$ is the only fixed point set of $h$ in $N(K)$. By Smith theory, $\operatorname{Fix}(h)=K$, which then contradicts the solution of the Smith Conjecture. 
Case 2. $h_{*}([m])=-[m] . h$ is now an orientation reversing homeomorphism. Since $h \circ h \in \operatorname{ker}(\sigma)$, and $h_{*} h_{*}([m])=[m]$, one has $h \circ h=$ id by the solution of Case 1 . Hence $h$ is an orientation reversing involution of $S^{3}$ with fixed point set containing $K$. Because the $\operatorname{Fix}(h)$ is a submanifold of odd codimension and contains $K$, $\operatorname{Fix}(h)$ contains a 2-manifold. By Smith Theory, the $\operatorname{Fix}(h)$ is a $Z_{2}$ homology sphere. Hence $\operatorname{Fix}(h)$ is a 2-sphere and contains $K$. This implies that $K$ is a trivial knot which is absurd.

Therefore $G$ is a subgroup of $\operatorname{Isom}(K, d)$. It is well known that a finite subgroup of $\operatorname{Isom}(K, d)$ is a cyclic or a dihedral group. In case $K$ is a hyperbolic knot, Out $\left(\pi_{1}\left(S^{3}-K\right)\right)$ acts isometrically on $S^{3}-\operatorname{int}(N(K))$ where $\partial N(K)$ is a flat torus in $S^{3}=K$ (see [M, B], or [Th]). Hence $\operatorname{Out}\left(\pi_{1}\left(S^{3}-K\right)\right.$ ) (or the same $\operatorname{Isom}\left(S^{3}-K\right)$ ) is a cyclic or a dihedral group.

Proof of Corollary 3. By Theorem 2 and its proof, the $Z_{n}$-action extends to a $Z_{n}$-action on $S^{3}$ such that $K$ is invariant and $K$ intersects the fixed point set of a nontrivial element $f$ in $Z_{n}$ if and only if $\operatorname{Fix}(\sigma(f)) \cap K \neq \varnothing$. But $\operatorname{Fix}(\sigma(f)) \cap K \neq \varnothing$ if and only if $\sigma(f)$ is a reflection on $K$ which in turn is the same as $f_{*}([l])=-[l]$ in $H_{1}(\partial N(K), Z)$. Moreover, in this case, $K$ intersects $\operatorname{Fix}(f)$ transversely in two points. The classification is now reduced to the classification of smooth cyclic group actions on $S^{3}$.

(I) The $Z_{n}$-action preserves the orientation.

If the $Z_{n}$-action on $S^{3}$ is fixed point free, we have (a). Otherwise, by Smith theory, the fixed point set is a knot, say $L$. The solution of the Smith Conjecture shows that $L$ is a trivial knot, and the $Z_{n}$-action is a $2 \pi / n$-rotation about $L$. Let $g$ be a generator of the $Z_{n}$-action. If $L$ intersects $K$, then by the remark above, we have $g_{*}([l])=[l]$, and $\sigma(g)$ is a reflection in $K$. Hence $\operatorname{Fix}(g \circ g)$ contains $K$. However $g g$ is orientation preserving. Therefore the solution of the Smith Conjecture implies that $g \circ g$ is the identity, i.e., $n=2$. This proves (b).

(II) The $Z_{n}$-action does not preserve the orientation.

Let $g$ still be the generator of the $Z_{n}$-action on $S^{3}$. Since $g$ reverses the orientation, $g$ has fixed points in $S^{3}, n$ is even, and Fix $(g)$ is a submanifold of odd codimension in $S^{3}$.

(c) $n=2$.

By Smith theory, $\operatorname{Fix}(g)$ is a $Z_{2}$-homology sphere. Hence $\operatorname{Fix}(g)$ is the two points set or the 2-sphere. If $\operatorname{Fix}(g)$ is the two points set, 
by Livesay's theorem [L], the $Z_{2}$-action is a reflection of $S^{3}$ through two points; if $\operatorname{Fix}(g)$ is a 2-sphere, then the action is a reflection of $S^{3}$ with respect to a 2 -sphere by Schonflies theorem. Now the $Z_{2}$-action is classified as follows. If $g_{*}([l])=[l]$, then $\operatorname{Fix}(g) \cap K=\varnothing$. In this case $\operatorname{Fix}(G)$ cannot be a 2 -sphere. To see this, $\operatorname{Fix}(g) \cap K=\varnothing$. In this case $\operatorname{Fix}(G)$ cannot be a 2-sphere. To see this, $\operatorname{Fix}(g) \cap K=\varnothing$ implies the fixed point set of $g$ in $S^{3}$ is actually in $S^{3}-\operatorname{int}(N(K))$. By Smith theory, for the $g$ involution on the one-dimensional homology sphere $S^{3}-\operatorname{int}(N(K)), \operatorname{Fix}\left(\left.g\right|_{S^{3}-\operatorname{int}(N(K))}\right)$ is a $Z_{2}$-homology sphere of dimension at most one. Hence $\operatorname{Fix}(g)$ are two points. This gives $(\mathrm{c})_{1}$. If $g_{*}([l])=-[l]$, then $\sigma(g)$ is a reflection in $K$, and $K$ intersects $\operatorname{Fix}(g)$ transversely in two points. $(c)_{2},(c)_{3}$ follow from the above mentioned classification of the orientation reversing involutions of $S^{3}$.

(d) $n \geq 4$.

The result is a consequence of the following proposition which will be proven in the appendix.

Proposition. Any smooth cyclic group action on $S^{3}$ which does not preserve the orientation is conjugate to a twisted rotation of $S^{3}$, or to a reflection of $S^{3}$ through two points.

Applying the proposition, we need only to check that $K$ is disjoint from the axis of the twisted rotation $g$. However the axis of $g$ is $\operatorname{Fix}(g \circ g)$. Fix $(g \circ g)$ does not intersect $K$ follows now from $g_{*} g_{*}([l])=[l]$, and $g \circ g \neq \mathrm{id}$. This completes the proof of $(\mathrm{d})$.

Corollary 4 is actually proven in the proof of Corollary 3.

Proof of Corollary 5. (a) By Proposition 3.19 of $[\mathbf{B}, \mathbf{Z}], K$ is invertible if and only if there is an automorphism

$$
\phi: \pi_{1}\left(S^{3}-\operatorname{int}(N(K))\right) \rightarrow \pi_{1}\left(S^{3}-\operatorname{int}(N(K))\right)
$$

such that $\phi(m)=m^{-1}$ and $\phi(l)=l^{-1}$. Since $K$ is a hyperbolic knot, Mostow Rigidity Theorem shows that $\phi$ can be realized by a hyperbolic isometry $h: S^{3}-\operatorname{int}(N(K)) \rightarrow S^{3}-\operatorname{int}(N(K))$ such that $h_{*}([m])=-[m]$, and $h_{*}([l])=-[l]$ in $H_{1}(\partial N(K), Z)$. Here we have assumed that $\partial N(K)$ is a flat torus in $S^{3}-K$. The condition $h_{*}([l])=-[l]$ implies that $h$ is an involution by Corollary 4. Because $h_{*}([m])=-[m], h$ is orientation preserving. Hence by Corollary 3, the $Z_{2}$-action generated by the extension of $h$ on $S^{3}$ is induced by a $\pi$-rotation of $S^{3}$ about an axis $L . H_{*}([l])=-[l]$ implies that 
$L$ intersects $K$ transversely in two points. Therefore $K$ is invariant under a $\pi$-rotation about an axis intersecting $K$ at two points. The inverse implication is trivial.

(b) By Proposition 3.19 of $[\mathbf{B}, \mathbf{Z}], K$ is amphicheiral if and only if there is an automorphism

$$
\phi: \pi_{1}\left(S^{3}-\operatorname{int}(N(K))\right) \rightarrow \pi_{1}\left(S^{3}-\operatorname{int}(N(K))\right)
$$

such that $\phi(m)=m^{-1}$ and $\phi(l)=l$. Realize $\phi$ by an isometry $h: S^{3}-\operatorname{int}(N(K)) \rightarrow S^{3}-\operatorname{int}(N(K)) . h$ is orientation reversing since $h_{*}([m])=-[m]$, and $h_{*}([l])=[l]$ in $H_{1}(\partial N(K), Z) . h$ generates a smooth cyclic group action on $S^{3}-\operatorname{int}(N(K))$ which does not preserve the orientation. Hence by Corollary $3, h$ is induced by a twisted rotation of $S^{3}$ about an axis $L$ missing $K$ if the order of $h$ is at least four. If the order of $h$ is two, the $h$ involution is the case (c) 1 in Corollary 3 because $h_{*}([l])=[l]$. Therefore, in this case $K$ is invariant under a reflection of $S^{3}$ through two points missing $K$. Then the condition is clearly sufficient.

(c) If the knot is both invertible and amphicheiral, then there exists an automorphism

$$
\phi: \pi_{1}\left(S^{3}-\operatorname{int}(N(K))\right) \rightarrow \pi_{1}\left(S^{3}-\operatorname{int}(N(K))\right)
$$

such that $\phi(m)=m$, and $\phi(l)=l^{-1} . \phi$ is the composition of the two automorphisms coming from (a) and (b). Realize $\phi$ by an orientation reversing hyperbolic isometry $h$ such that $h_{*}([m])=[m]$, and $h_{*}([l])=-[l]$ in $H_{1}(\partial N(K), Z)$. By Corollary $4, h_{*}([l])=-[l]$ and $h_{*}([\mathrm{~m}])=[\mathrm{m}]$ imply $h$ is an orientation reversing involution of $S^{3}-\operatorname{int}(N(K)) \rightarrow S^{3}-N(K)$. By Corollary $3, h$ is the case (c) 2 or the case $(\mathrm{c})_{3}$. Case (c) $)_{3}$ cannot happen since $K$ is a prime knot. Hence $K$ is invariant under the reflection of $S^{3}$ through two points contained in $K$.

Appendix. We prove the following proposition concerning smooth cyclic group action on the 3-sphere which does not preserve the orientation.

Proposition. Any smooth non-orientation preserving cyclic group action on $S^{3}$ is conjugate to a twisted rotation of $S^{3}$, or to a reflection of $S^{3}$ through two points.

Proof. Let $g$ be a generator of the $Z_{n}$-action. $n$ has to be even. $g$ is orientation reversing, and hence has fixed points in $S^{3}$. If $n=2$, 
we have shown in the proof of Corollary 3 (c) that the result holds. Assume $n \geq 4$ from now on. Let $h=g \circ g$. $h$ is an orientation preserving automorphism of order $m$, and has fixed points. The solution of the Smith Conjecture shows that the $\operatorname{Fix}(h)$ is a trivial knot, say $L$. Now $L$ is invariant under $g . g$ acts on $L$ with fixed point and is of order two in $L$. Hence the action of $g$ on $L$ is a reflection by the classification of $Z_{2}$-action on the circle. Take a $Z_{n^{-}}$equivariant regular neighborhood $N(L)$ of $L$ in $S^{3}$ (see [B]). By the choice of the regular neighborhood, one knows that the action of $Z_{n}$ on $N(L)$ is standard. Therefore by choosing the generator $g$ of the $Z_{n}$-action appropriately, we can assume that the restriction of $g$ on $N(L)=D^{2} \times S^{1}$ is conjugate to $\alpha$, where

$$
\alpha: D^{2} \times S^{1} \rightarrow D^{2} \times S^{1}
$$

sends $(z, w)$ to $\left(e^{2 \pi i / n} z, \bar{w}\right)$, with $z$ in $D^{2}=\{z \in C|| z \mid \leq 1\}$ and $w$ in $S^{1}=\{z \in C|| z \mid=1\}$. Note that $\alpha$ generates an orientation reversing $Z_{n}$-action on $D^{2} \times S^{1}$ with two fixed points in $\{0\} \times S^{1}$. Since $L$ is the trivial knot, $S^{3}-\operatorname{int}(N(L))$ is a solid torus. Let $\phi: S^{3}=\left(S^{3}-\operatorname{int}(N(L))\right) \cup N(L) \rightarrow \bar{S}^{3}=\left(S^{1} \times D^{2}\right) \cup_{\text {id }}\left(D^{2} \times S^{1}\right)$ be a diffeomorphism taking $N(L)$ to $D^{2} \times S^{1}$ such that $\left.\phi g\right|_{N(L)} \phi^{-1}=$ $\alpha$. Now extend $\alpha$ to be a self-diffeomorphism $\bar{\alpha}$ of $\bar{S}^{3}$ by sending $(z, w) S^{1} \times D^{2}$ to $\left(e^{2 \pi i / n} z, \bar{w}\right)$ with $z \in S^{1}$ and $w \in D^{2}$. Then $\bar{\alpha}$ generates a twisted $2 \pi / n$-rotation of $\bar{S}^{3}$. Our goal is to show that $\phi g \phi^{-1}$ is conjugate to $\bar{\alpha}$ in $\bar{S}^{3}$. This is consequence of the following claim.

Claim. $g^{\prime}=\left.\phi g \phi^{-1}\right|_{S^{1} \times D^{2}}$ is conjugate to $\beta=\left.\bar{\alpha}\right|_{S^{1} \times D^{2}}$ by a piecewise smooth diffeomorphism $\psi$ such that $\psi$ is the identity map on $\partial\left(S^{1} \times D^{2}\right)$.

Let us assume the claim and finish the proof. By gluing $\psi$ with Id $\left.\right|_{D^{2} \times S^{1}}$ along the boundaries, we obtain a piecewise smooth selfdiffeomorphism of $\bar{S}^{3}$ which conjugates $\phi g \phi^{-1}$ to $\bar{\alpha}$. Therefore $\phi g \phi^{-1}$ is smoothly conjugate to $\bar{\alpha}$ by the work of Moise.

Proof of the Claim. By the choice of $\phi, g^{\prime}$ is the same as $\beta$ on $\partial\left(S^{1} \times D^{2}\right)$. Using the equivariant Dehn's lemma, we can find $n$ copies of disjoint properly embedded disks $D_{1}, D_{2}, \ldots, D_{n}$ with $\partial D_{j}$ $=e^{e \pi j i / n} \times \partial D^{2}$ in $S^{1} \times D^{2}$, such that $g^{\prime}\left(D_{j}\right)=D_{j+1}$ for $j=$ 
$1,2, \ldots, n$, where $D_{1}=D_{n+1} \cdot g^{\prime}: D_{j} \rightarrow D_{j+1}$ is a diffeomorphism for each $j$. These disks cut $S^{1} \times D^{2}$ into $n$ components, say $B_{1}, B_{2}, \ldots, B_{n}$ with $D_{j} \cup D_{j+1} \subset \partial B_{j}$, and each of $B_{j}$ is a 3-ball by Schonflies' theorem. Let $D_{j}^{\prime}=e^{2 \pi i j / n} \times D^{2}$ (where $\left.D_{n+1}^{\prime}=D_{1}^{\prime}\right)$; $B_{j}^{\prime}=\left\{e^{2 \pi i t / n} \mid j \leq t \leq j+1\right\} \times D^{2}$; and $E_{j}=\partial B_{j}^{\prime}-\left(D_{j} \cup D_{j+1}\right)$, the annulus, for each $i=1,2, \ldots, n$. The construction of $\psi$ is now as follows. Let $A_{1}: D_{1} \rightarrow D_{1}^{\prime}$ be a diffeomorphism which is the identity on $\partial D_{1}$. Define $A_{2}: D_{2} \rightarrow D_{2}^{\prime}$ to be $\left.\left.\beta\right|_{D_{1}^{\prime}} A_{1} g^{\prime-1}\right|_{D_{2}}$. It is still a diffeomorphism which fixes $\partial D_{2}$ pointwise. Since $\partial B_{1}=D_{1} \cup E_{1} \cup D_{2}$ and $\partial B_{1}^{\prime}=D_{1}^{\prime} \cup E_{1} \cup D_{2}^{\prime}$, glue $A_{1}, A_{2}$ and $\left.\mathrm{id}\right|_{E_{1}}$ along the boundaries, one obtains a piecewise smooth diffeomorphism from $\partial B_{1} \rightarrow \partial B_{1}^{\prime}$ which is the identity on $E_{1}$. Extend it to be a piecewise smooth diffeomorphism from $B_{1}$ to $B_{1}^{\prime}$ by Alexander's lemma, and call it $\psi_{1}$. Now $\psi_{j}: B_{j} \rightarrow B_{j}^{\prime}$ is defined to be

$$
\left.\left.\beta_{j}\right|_{B_{1}^{\prime}} \psi_{1} g^{\prime-j}\right|_{B_{j}}
$$

for $j=2,3, \ldots, n$. All these piecewise smooth diffeomorphisms match on the $D_{j}$ 's. Gluing them together along the $D_{j}$ 's, we obtain a piecewise diffeomorphism $\psi: S^{1} \times D^{2} \rightarrow S^{1} \times D^{2}$. Then $\left.\psi\right|_{\partial\left(S^{1} \times D^{2}\right)}=$ id and $\beta=\psi^{-1} \beta \psi$.

\section{REFERENCES}

[B] G. Bredon, Introduction to Compact Transformation Groups, Academic Press, New York (1972).

[B,S] S. Bleiler and M. Scharlemann, Strongly invertible knots have property $P$, preprint.

[B,Z] G. Burde and H. Zieschang, Knots, Walter de Gruyter, Berlin-New York (1985).

[CGLS] M. Culler, C. Gordon, J. Luecke, and P. Shalen, Dehn surgery on knots, MSRI preprint.

[G,L] M. Gordon and C. Luecke, Knots are determined by their complements, Bull. Amer. Math. Soc., 20, No. 1, (1989), 83-84.

[E] A. Edmonds, Transformation Groups and Low-Dimensional Manifolds, Contemporary Math., 36 (1985), 339-366.

[G] D. Gabai, Surgery on knots in solid tori, preprint.

[Ka] A. Kawauchi, The invertibility problem on amphicheiral excellent knots, Proc. Japan Acad., 55 (1979), 399-402.

[K] B. Kirby, Problems in Low-Dimensional Topology, Proc. Sympos. Pure Math., Amer. Math. Soc., (1978).

[L] G. Livesay, Involutions with two fixed points on the three-sphere, Ann. of Math., 78 (1963), 582-593.

[M,B] J. Morgan and H. Bass, The Smith Conjecture, Academic Press, New York (1982). 
[Ri] R. Riley, Seven Excellent Knots, Proceedings of the Conference on Topology in Low Dimension, 81-151, Cambridge University Press, Cambridge (1982).

[R] D. Rolfson, Knots and Links, Publish or Perish, Inc., Berkeley (1976).

[Th] W. Thurston, The Geometry and Topology of 3-Manifold, Lecture Notes, Dept. of Math., Princeton Univ., Princeton, NJ (1978).

Received February 23, 1991. Supported in part by NSF DMS 86-3126.

UNIVERSITY OF CALIFORNIA

LOS ANGELES, CA 90024-1555 



\section{PACIFIC JOURNAL OF MATHEMATICS EDITORS}

\author{
V. S. VARADARAJAN \\ (Managing Editor) \\ University of California \\ Los Angeles, CA 90024-1555 \\ Herbert Clemens \\ University of Utah \\ Salt Lake City, UT 84112 \\ F. Michael Christ \\ University of California \\ Los Angeles, CA 90024-1555 \\ THOMAS ENRIGHT \\ University of California, San Diego \\ La Jolla, CA 92093
}

\author{
Nicholas ERcolani \\ University of Arizona \\ Tucson, AZ 85721 \\ R. FINN \\ Stanford University \\ Stanford, CA 94305 \\ VAughan F. R. JONES \\ University of California \\ Berkeley, CA 94720 \\ C. C. Moore \\ University of California \\ Berkeley, CA 94720 \\ MARTIN ScharlemanN \\ University of California \\ Santa Barbara, CA 93106 \\ Harold Stark \\ University of California, San Diego \\ La Jolla, CA 92093
}

STEVEN KeRCKHOFF

Stanford University

Stanford, CA 94305

\begin{tabular}{|c|c|c|c|c|}
\hline \multicolumn{5}{|c|}{ ASSOCIATE EDITORS } \\
\hline R. Arens & $\begin{array}{ll}\text { E. F. BECKENBACH } & \text { B. H } \\
(1906-1982) & \end{array}$ & NeUmanN & $\begin{array}{c}\text { F. WoLF } \\
(1904-1989)\end{array}$ & K. Yoshida \\
\hline \multicolumn{5}{|c|}{ SUPPORTING INSTITUTIONS } \\
\hline \multicolumn{2}{|c|}{ UNIVERSITY OF ARIZONA } & \multicolumn{3}{|c|}{ UNIVERSITY OF OREGON } \\
\hline \multicolumn{2}{|c|}{ UNIVERSITY OF BRITISH COLUMBIA } & \multicolumn{3}{|c|}{ UNIVERSITY OF SOUTHERN CALIFORNIA } \\
\hline \multicolumn{2}{|c|}{ CALIFORNIA INSTITUTE OF TECHNOLOGY } & \multicolumn{3}{|c|}{ STANFORD UNIVERSITY } \\
\hline \multicolumn{2}{|c|}{ UNIVERSITY OF CALIFORNIA } & \multicolumn{3}{|c|}{ UNIVERSITY OF HAWAII } \\
\hline \multicolumn{2}{|c|}{ MONTANA STATE UNIVERSITY } & \multicolumn{3}{|c|}{ UNIVERSITY OF TOKYO } \\
\hline \multicolumn{2}{|c|}{ UNIVERSITY OF NEVADA, RENO } & \multicolumn{3}{|c|}{ UNIVERSITY OF UTAH } \\
\hline & \multicolumn{3}{|c|}{ WASHINGTON STATE UNIVERSITY } \\
\hline \multicolumn{2}{|c|}{ OREGON STATE UNIVERSITY } & \multicolumn{3}{|c|}{ UNIVERSITY OF WASHINGTON } \\
\hline
\end{tabular}

The Supporting Institutions listed above contribute to the cost of publication of this Journal, but they are not owners or publishers and have no responsibility for its content or policies.

Mathematical papers intended for publication in the Pacific Journal of Mathematics should be in typed
form or offset-reproduced (not dittoed), double spaced with large margins. Please do not use built up fractions
in the text of the manuscript. However, you may use them in the displayed equations. Underline Greek letters
in red, German in green, and script in blue. The first paragraph must be capable of being used separately as
a synopsis of the entire paper. In particular it should contain no bibliographic references. Please propose a
heading for the odd numbered pages of less than 35 characters. Manuscripts, in triplicate, may be sent to any
one of the editors. Please classify according to the 1991 Mathematics Subject Classification scheme which
can be found in the December index volumes of Mathematical Reviews. Supply name and address of author
to whom proofs should be sent. All other communications should be addressed to the managing editor, or
Elaine Barth, University of California, Los Angeles, California $90024-1555-05$.
There are page-charges associated with articles appearing in the Pacific Journal of Mathematics. These
charges are expected to be paid by the author's University, Government Agency or Company. If the author or
authors do not have access to such Institutional support these charges are waived. Single authors will receive
50 free reprints; joint authors will receive a total of 100 free reprints. Additional copies may be obtained at
cost in multiples of 50 .

The Pacific Journal of Mathematics (ISSN 0030-8730) is published monthly except for July and August. Regular subscription rate: $\$ 190.00$ a year (10 issues). Special rate: $\$ 95.00$ a year to individual members of supporting institutions.

Subscriptions, orders for numbers issued in the last three calendar years, and changes of address should be sent to Pacific Journal of Mathematics, P.O. Box 969, Carmel Valley, CA 93924, U.S.A. Old back numbers obtainable from Kraus Periodicals Co., Route 100, Millwood, NY 10546.

The Pacific Journal of Mathematics at P.O. Box 969, Carmel Valley, CA 93924 (ISSN 0030-8730) is published monthly except for July and August. Second-class postage paid at Carmel Valley, California 93924, and additional mailing offices. Postmaster: send address changes to Pacific Journal of Mathematics, P.O. Box 969, Carmel Valley, CA 93924.

PUBLISHED BY PACIFIC JOURNAL OF MATHEMATICS, A NON-PROFIT CORPORATION Copyright (C) 1992 by Pacific Journal of Mathematics 


\section{Pacific Journal of Mathematics}

\section{Vol. 154, No. $2 \quad$ June, 1992}

Manuel (Rodriguez) de León, J. A. Oubiña, P. R. Rodrigues and

Modesto R. Salgado, Almost $s$-tangent manifolds of higher order . . . . 201

Martin Engman, New spectral characterization theorems for $S^{2} \ldots \ldots \ldots 215$

Yuval Zvi Flicker, The adjoint representation $L$-function for GL $(n) \ldots \ldots 231$

Enrique Alberto Gonzalez-Velasco and Lee Kenneth Jones, On the range

of an unbounded partly atomic vector-valued measure . ............ 245

Takayuki Hibi, Face number inequalities for matroid complexes and

Cohen-Macaulay types of Stanley-Reisner rings of distributive

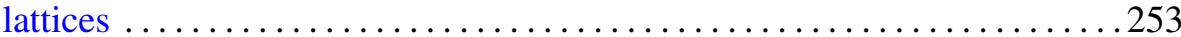

Hervé Jacquet and Stephen James Rallis, Kloosterman integrals for skew

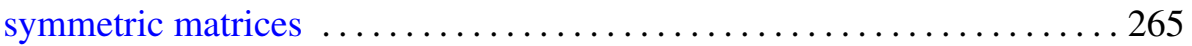

Shulim Kaliman, Two remarks on polynomials in two variables .........285

Kirk Lancaster, Qualitative behavior of solutions of elliptic free boundary problems ..........................................297

Feng Luo, Actions of finite groups on knot complements . ........... 317

James Joseph Madden and Charles Madison Stanton, One-dimensional

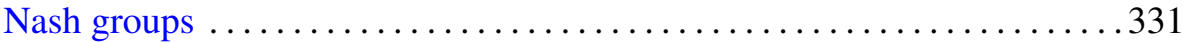

Christopher K. McCord, Estimating Nielsen numbers on

infrasolvmanifolds ......................................... 345

Gordan Savin, On the tensor product of theta representations of $\mathrm{GL}_{3}$ 369

Gerold Wagner, On means of distances on the surface of a sphere. II.

(Upper bounds) 